\title{
Relativistic $x$-ray free-electron lasers in the quantum regime
}

\author{
Bengt Eliasson ${ }^{1}$ and P. K. Shukla ${ }^{2,3}$ \\ ${ }^{1}$ Institut für Theoretische Physik, Fakultät für Physik und Astronomie, Ruhr-Universität Bochum, D-44780 Bochum, Germany \\ ${ }^{2}$ International Centre for Advanced Studies in Physical Sciences \& Institute for Theoretical Physics, Fakultät für Physik und Astronomie, \\ Ruhr-Universität Bochum, D-44780 Bochum, Germany \\ ${ }^{3}$ Department of Mechanical and Aerospace Engineering \& Center for Energy Research, University of California San Diego, \\ La Jolla, California 92093, USA
}

(Received 15 December 2011; published 20 June 2012)

\begin{abstract}
We present a nonlinear theory for relativistic x-ray free-electron lasers in the quantum regime, using a collective Klein-Gordon (KG) equation (for relativistic electrons), which is coupled with the Maxwell-Poisson equations for the electromagnetic and electrostatic fields. In our model, an intense electromagnetic wave is used as a wiggler which interacts with a relativistic electron beam to produce coherent tunable radiation. The KG-Maxwell-Poisson model is used to derive a general nonlinear dispersion relation for parametric instabilities in three space dimensions, including an arbitrarily large amplitude electromagnetic wiggler field. The nonlinear dispersion relation reveals the importance of quantum recoil effects and oblique scattering of the radiation that can be tuned by varying the beam energy.
\end{abstract}

DOI: 10.1103/PhysRevE.85.065401

PACS number(s): 52.35.Mw, 41.60.Cr, 52.38.Hb, 52.40.Db

Introduction. With the recent development of $\mathrm{x}$-ray freeelectron lasers (FELs) [1] there are new possibilities to explore matter on atomic and single molecule levels. On these length scales, of the order of a few angstroms, quantum effects play an important role in the dynamics of the electrons. Quantum effects have been measured experimentally both in the degenerate electron gas in metals and in warm dense matter [2], and must also be taken into account in intense laser-solid density plasma interaction experiments [3]. The theory of the FEL was originally developed in the framework of quantum mechanics [4], but where Planck's constant canceled out in the final FEL gain formula, producing a classical result. It was subsequently shown that classical theory can be used and quantum effects can be neglected [5], if the photon momentum recoil is not larger than the beam momentum spread [6-9]. To overcome the technical limitation on the wiggler wavelength for a static magnetic field wiggler, it was suggested that it can be replaced by an electromagnetic (EM) wiggler or by a plasma wave wiggler [10] to generate short wavelength radiation. In such a situation, it turns out that quantum recoil effects can be important. The Klein-Gordon equation (KGE) for a single electron was used to derive a general set of quantum mechanical equations for the FEL [11], while a single-electron Schrödinger-like equation for the dynamics of the FEL was derived using the field theory [12]. The nonlinear quantum regime of $\mathrm{x}$-ray Compton lasers have been investigated using Volkov-dressed electrons based on the Dirac equation [13]. Furthermore, by using a multielectron FEL Hamiltonian, it was shown that quantum effects can lead to the splitting of the radiated spectrum into narrow bands for short electron bunches $[14,15]$. Relativistic and collective quantum effects have been studied for FELs using Wigner $[16,17]$ and quantum fluid [18] models.

In this Rapid Communication, we shall use a collective KGE to derive a dynamic model for the quantum free-electron laser. In our model, we assume that the wave function $\psi$ represents an ensemble of electrons, so that the resulting charge and current densities act as sources [19] for the self-consistent electrodynamic vector and scalar potentials A and $\phi$, respectively.

Mathematical model. The KGE in the presence of the electrodynamic fields reads

$$
\mathcal{W}^{2} \psi-c^{2} \mathcal{P}^{2} \psi-m_{e}^{2} c^{4} \psi=0,
$$

where the energy and momentum operators are $\mathcal{W}=i \hbar \partial / \partial t+$ $e \phi$ and $\mathcal{P}=-i \hbar \nabla+e \mathbf{A}$, respectively. Here $\hbar$ is Planck's constant divided by $2 \pi, e$ the magnitude of the electron charge, $m_{e}$ the electron rest mass, and $c$ the speed of light in vacuum. The electrodynamic potentials are obtained self-consistently from the Maxwell equations

$$
\frac{\partial^{2} \mathbf{A}}{\partial t^{2}}+c^{2} \nabla \times(\nabla \times \mathbf{A})+\nabla \frac{\partial \phi}{\partial t}=\mu_{0} c^{2} \mathbf{j}_{e}
$$

and

$$
\nabla^{2} \phi+\nabla \cdot \frac{\partial \mathbf{A}}{\partial t}=-\frac{1}{\varepsilon_{0}}\left(\rho_{e}+\rho_{i}\right)
$$

where $\mu_{0}$ is the magnetic permeability, $\varepsilon_{0}=1 / \mu_{0} c^{2}$ the electric permittivity in vacuum, and $\rho_{i}=e n_{0}$ a neutralizing positive charge density due to ions, where $n_{0}$ is the equilibrium electron number density. The electric charge and current densities of the electrons are $\rho_{e}=-e\left[\psi^{*} \mathcal{W} \psi+\psi(\mathcal{W} \psi)^{*}\right] / 2 m_{e} c^{2}$ and $\mathbf{j}_{e}=-e\left[\psi^{*} \mathcal{P} \psi+\psi(\mathcal{P} \psi)^{*}\right] / 2 m_{e}$, respectively. They obey the continuity equation $\partial \rho_{e} / \partial t+\nabla \cdot \mathbf{j}_{e}=0$.

The growth rate of the scattering instability is calculated in the beam frame and the result is then Lorentz transformed into the laboratory frame. We assume that the beam is propagating along the $z$ axis, in the opposite direction of the laser wiggler beam. For the laser wiggler field, we consider for simplicity a circularly polarized EM wave of the form $\mathbf{A}_{0}=(1 / 2) \widehat{\mathbf{A}}_{0} \exp \left(-i \omega_{0} t+i \mathbf{k}_{0} \cdot \mathbf{r}\right)+$ complex conjugate, with $\widehat{\mathbf{A}}_{0}=(\widehat{\mathbf{x}}+i \widehat{\mathbf{y}}) \widehat{A}_{0}$, where $\omega_{0}$ is the laser wave frequency and $\mathbf{k}_{0}=k_{0} \widehat{\mathbf{z}}$ the wave vector, and $\widehat{\mathbf{x}}, \widehat{\mathbf{y}}$, and $\widehat{\mathbf{z}}$ the unit vectors in the $x, y$, and $z$ directions, respectively. Due to the circular polarization, the oscillatory parts in the nonlinear term proportional to $A^{2}$ in the KGE vanish. Hence, our starting 
point is the nonlinear dispersion relation in the beam frame, with primed variables, where the plasma is at rest. It reads [20]

$$
1-\frac{\left(\omega_{p e}^{\prime}\right)^{2}}{4 \gamma_{A}^{3} m_{e}^{2} c^{2}} \frac{D_{A}^{\prime}\left(\Omega^{\prime}, \mathbf{K}^{\prime}\right)}{D_{L}^{\prime}\left(\Omega^{\prime}, \mathbf{K}^{\prime}\right)} \sum_{+,-} \frac{e^{2}\left|\mathbf{k}_{ \pm}^{\prime} \times \widehat{\mathbf{A}}_{0}^{\prime}\right|^{2}}{\left(k_{ \pm}^{\prime}\right)^{2} D_{A}^{\prime}\left(\omega_{ \pm}^{\prime}, \mathbf{k}_{ \pm}^{\prime}\right)}=0
$$

where the electron plasma oscillations in the presence of the EM field are represented by

$$
\begin{aligned}
D_{L}^{\prime}\left(\Omega^{\prime}, \mathbf{K}^{\prime}\right)= & \frac{\left(\omega_{p e}^{\prime}\right)^{2}}{\gamma_{A}}-\left(\Omega^{\prime}\right)^{2} \\
& +\frac{\hbar^{2}\left[c^{2}\left(K^{\prime}\right)^{2}-\left(\Omega^{\prime}\right)^{2}\right]}{4 \gamma_{A}^{2} m_{e}^{2} c^{4}} D_{A}^{\prime}\left(\Omega^{\prime}, \mathbf{K}^{\prime}\right) .
\end{aligned}
$$

Here $\Omega^{\prime}$ and $\mathbf{K}^{\prime}$ are the frequency and wave vector of the plasma oscillations, respectively, $\gamma_{A}=\left(1+a_{0}^{2}\right)^{1 / 2}$ is the relativistic gamma factor due to the large amplitude EM field, and $a_{0}=e\left|\widehat{A}_{0}^{\prime}\right| / m_{e} c$ is the normalized amplitude of the EM wave (the wiggler parameter). The dispersion relation for the beam oscillations in the presence of a large amplitude EM wave is given by $D_{L}^{\prime}\left(\Omega^{\prime}, \mathbf{K}^{\prime}\right)=0$. The EM sidebands are governed by

$$
D_{A}^{\prime}\left(\omega_{ \pm}^{\prime}, \mathbf{k}_{ \pm}^{\prime}\right)=c^{2}\left(k_{ \pm}^{\prime}\right)^{2}-\left(\omega_{ \pm}^{\prime}\right)^{2}+\left(\omega_{p e}^{\prime}\right)^{2} / \gamma_{A},
$$

where $\omega_{ \pm}^{\prime}=\omega_{0}^{\prime} \pm \Omega^{\prime}$ and $\mathbf{k}_{ \pm}^{\prime}=\mathbf{k}_{0}^{\prime} \pm \mathbf{K}^{\prime}$, and $\omega_{0}^{\prime}$ and $k_{0}^{\prime}$ are related through the nonlinear dispersion relation $\left(\omega_{0}^{\prime}\right)^{2}=$ $c^{2}\left(k_{0}^{\prime}\right)^{2}+\left(\omega_{p e}^{\prime}\right)^{2} / \gamma_{A}$. We also denoted $D_{A}^{\prime}(\Omega, \mathbf{K})=c^{2}\left(K^{\prime}\right)^{2}-$ $\left(\Omega^{\prime}\right)^{2}+\left(\omega_{p e}^{\prime}\right)^{2} / \gamma_{A}$. We have neglected the two-plasmon decay [21], which would give rise to terms proportional to $\left|\mathbf{k}_{ \pm}^{\prime} \cdot \widehat{\mathbf{A}}_{0}^{\prime}\right|^{2}$ in Eq. (4).

To move from the beam frame to the laboratory frame, the time and space variables are Lorentz transformed as $t^{\prime}=\gamma_{0}\left(t-v_{0} z / c^{2}\right), x^{\prime}=x, y^{\prime}=y$, and $z^{\prime}=\gamma_{0}\left(z-v_{0} t\right)$, where $\mathbf{v}_{0}=v_{0} \widehat{\mathbf{z}}$ is the beam velocity, and $\gamma_{0}=1 / \sqrt{1-v_{0}^{2} / c^{2}}$ the gamma factor due to the relativistic beam speed. The corresponding frequency and wave-number transformations are thus $\omega^{\prime}=\gamma_{0}\left(\omega-v_{0} k_{z}\right), k_{x}^{\prime}=k_{x}, k_{y}^{\prime}=k_{y}$, and $k_{z}^{\prime}=$ $\gamma_{0}\left(k_{z}-v_{0} \omega / c^{2}\right)$, They apply to the frequency and wave vector pairs $(\Omega, \mathbf{K}),\left(\omega_{0}, \mathbf{k}_{0}\right)$, and $\left(\omega_{ \pm}, \mathbf{k}_{ \pm}\right)$. The plasma frequency is transformed as $\omega_{p e}^{\prime}=\omega_{p e} \sqrt{\gamma_{A} / \gamma}$, where $\gamma=$ $\left(1+p_{0}^{2} / m_{e}^{2} c^{2}+a_{0}^{2}\right)^{1 / 2}$ is the total gamma factor and $\mathbf{p}_{0}=$ $\gamma m_{e} \mathbf{v}_{0}$ the relativistic electron momentum. Since the components of $\widehat{\mathbf{A}}_{0}$ are perpendicular to the beam velocity direction, they are not affected by the Lorentz transformation, hence $\widehat{\mathbf{A}}_{0}^{\prime}=\widehat{\mathbf{A}}_{0}$. We also use the relation $\gamma_{A} \gamma_{0}=\gamma$, and observe that the expressions of the form $\left(\omega^{\prime}\right)^{2}-c^{2}\left(k^{\prime}\right)^{2}=\omega^{2}-c^{2} k^{2}$ are Lorentz invariant. This yields $D_{L}^{\prime}\left(\Omega^{\prime}, \mathbf{K}^{\prime}\right)=\gamma_{0}^{2} D_{L}(\Omega, \mathbf{K})$, with

$$
\begin{aligned}
D_{L}(\Omega, \mathbf{K})= & \frac{\omega_{p e}^{2} \gamma_{A}^{2}}{\gamma^{3}}-\left(\Omega-v_{0} K_{z}\right)^{2} \\
& +\frac{\hbar^{2}\left(c^{2} K^{2}-\Omega^{2}\right)}{4 \gamma^{2} m_{e}^{2} c^{4}} D_{A}(\Omega, \mathbf{K}),
\end{aligned}
$$

and $D_{A}^{\prime}\left(\omega_{ \pm}^{\prime}, \mathbf{k}_{ \pm}^{\prime}\right)=D_{A}\left(\omega_{ \pm}, \mathbf{k}_{ \pm}\right) \equiv c^{2} k_{ \pm}^{2}-\omega_{ \pm}^{2}+\omega_{p e}^{2} / \gamma$, and, similarly, $D_{A}^{\prime}\left(\Omega^{\prime}, \mathbf{K}^{\prime}\right)=D_{A}(\Omega, \mathbf{K})$. In the laboratory frame, Eq. (4) is of the form

$$
1-\frac{\omega_{p e}^{2}}{4 \gamma^{3} m_{e}^{2} c^{2}} \frac{D_{A}(\Omega, \mathbf{K})}{D_{L}(\Omega, \mathbf{K})} \sum_{+,-} \frac{e^{2}\left|\mathbf{k}_{ \pm}^{\prime} \times \widehat{\mathbf{A}}_{0}\right|^{2}}{\left(k_{ \pm}^{\prime}\right)^{2} D_{A}\left(\omega_{ \pm}, \mathbf{k}_{ \pm}\right)}=0 .
$$

Using $\mathbf{K}=K_{x} \widehat{\mathbf{x}}+K_{y} \widehat{\mathbf{y}}+K_{z} \widehat{\mathbf{z}}$, we have $K^{2}=K_{z}^{2}+K_{\perp}^{2}$ with $K_{\perp}^{2}=K_{x}^{2}+K_{y}^{2}$, so that $\left|\mathbf{k}_{ \pm}^{\prime} \times \widehat{\mathbf{A}}_{0}\right|^{2}=\left\{2 \gamma_{0}^{2}\left[k_{0} \pm K_{z}+\right.\right.$ $\left.\left.\left(v_{0} / c^{2}\right)\left(\omega_{0} \pm \Omega\right)\right]^{2}+K_{\perp}^{2}\right\}\left|\widehat{A}_{0}\right|^{2}$, and $\left(k_{ \pm}^{\prime}\right)^{2}=\gamma_{0}^{2}\left[k_{0} \pm K_{z}+\right.$ $\left.\left(v_{0} / c^{2}\right)\left(\omega_{0} \pm \Omega\right)\right]^{2}+K_{\perp}^{2}$.

For the resonant backscattering instability, we have $\left|D_{A}\left(\omega_{+}, \mathbf{k}_{+}\right)\right| \gg\left|D_{A}\left(\omega_{-}, \mathbf{k}_{-}\right)\right|$. Also, for $\Omega \approx v_{0} K_{z}, v_{0} \approx-c$, and $\gamma_{0} K_{z} \gg k_{0}, K_{\perp}$, we have $\left|\mathbf{k}_{ \pm}^{\prime} \times \widehat{\mathbf{A}}_{0}\right|^{2} /\left(k_{ \pm}^{\prime}\right)^{2} \approx 2\left|\widehat{A}_{0}\right|^{2}$. In this limit, Eq. (8) is written as

$$
D_{L}(\Omega, \mathbf{K}) D_{A}\left(\omega_{-}, \mathbf{k}_{-}\right)=\frac{\omega_{p e}^{2} D_{A}(\Omega, \mathbf{K})}{2 \gamma^{3}} a_{0}^{2} .
$$

By using $\Omega \approx v_{0} K_{z}$, the expression for $D_{L}(\Omega, \mathbf{K})$ can be simplified as

$$
D_{L}(\Omega, \mathbf{K})=\Omega_{p}^{2}-\left(\Omega-v_{0} K_{z}\right)^{2}
$$

where

$$
\Omega_{p}=\sqrt{\frac{\omega_{p e}^{2} \gamma_{A}^{2}}{\gamma^{3}}+\frac{\hbar^{2}\left(K_{z}^{2} \gamma_{A}^{2}+K_{\perp}^{2} \gamma^{2}\right)^{2}}{4 \gamma^{6} m_{e}^{2}}} .
$$

Equation (10) is valid for $\hbar \omega_{p e} \gamma_{A} / \gamma^{3 / 2} m_{e} c^{2} \ll 1$ (which is always fulfilled), and $\omega_{p e} \gamma_{A} /\left|v_{0}\right| \gamma^{3 / 2} \ll K_{z} \ll$ $m_{e}\left|v_{0}\right| \gamma^{3 / 2} / \hbar \gamma_{A}$. The latter condition, with $\left|v_{0}\right| \approx c$, gives $\gamma_{A} / \lambda_{e} \gamma^{3 / 2} \ll K_{z} \ll \gamma^{3 / 2} / \lambda_{C} \gamma_{A}$, where $\lambda_{e}=c / \omega_{p e}$ is the electron skin depth and $\lambda_{C}=\hbar / m_{e} c$ the reduced Compton length. We note that $\Omega_{p}$ contains a combination of the collective beam plasma oscillation and quantum recoil effects, which lead to a splitting of the beam mode into one slightly upshifted and one downshifted mode.

The resonant $\Omega$ and $\mathbf{K}$ are obtained by simultaneously setting $D_{L}(\Omega, \mathbf{K})=0$ and $D_{A}\left(\omega_{-}, \mathbf{k}_{-}\right)=0$. Invoking the approximation $D_{L}(\Omega, \mathbf{K}) \simeq-\left(\Omega-v_{0} K_{z}\right)^{2}=0$ and $D_{A}\left(\omega_{-}, \mathbf{k}_{-}\right) \simeq$ $c^{2}\left(k_{0}-K_{z}\right)^{2}+c^{2} K_{\perp}^{2}-\left(\omega_{0}-\Omega\right)^{2}=0$, we obtain $\Omega=v_{0} K_{z}$ and the resonance condition $\left(K_{z}-2 \gamma_{0}^{2} k_{0}\right)^{2}+\gamma_{0}^{2} K_{\perp}^{2}=4 \gamma_{0}^{4} k_{0}^{2}$ for $\omega_{0} \approx c k_{0}$ and $v_{0} \approx-c$. The corresponding resonant wave vector components of the radiation field, $\mathbf{k}_{-}=\mathbf{k}_{0}-\mathbf{K}$, shown in top panels of Fig. 3, form an ellipsoid in wave vector space rotationally symmetric around the $k_{z-}$ axis. The resulting radiation frequency $\omega_{-}=\omega_{0}-v_{0} K_{z} \simeq \omega_{0}+c K_{z}$ is strongly upshifted in the parallel direction $\left(K_{\perp}=0\right)$, where we have $K_{z}=4 \gamma^{2} k_{0} / \gamma_{A}^{2}$ and $\omega_{-} \approx 4 \gamma^{2} c k_{0} / \gamma_{A}^{2}$. The result differs by a factor two when compared with the case involving a static wiggler [22].

Comparing the two terms under the square root in Eq. (11), we see that the quantum recoil effect starts to be important in the parallel direction $\left(K_{\perp}=0, K_{z} \approx 4 \gamma^{2} k_{0} / \gamma_{A}^{2}\right)$ when $k_{0} \approx$ $k_{0 \text {,crit }}$, where

$$
k_{0, \text { crit }}=\left(\frac{\omega_{p e} \gamma_{A}^{3} m_{e}}{8 \hbar \gamma^{5 / 2}}\right)^{1 / 2} .
$$

In the classical limit $k_{0} \ll k_{0 \text {,crit }}$ (corresponding to the Raman regime discussed below), we have $\Omega_{p}=\omega_{p e} \gamma_{A} / \gamma^{3 / 2}$, while for $k_{0} \gg k_{0 \text {,crit }}$, the quantum effects dominate and we have $\Omega_{p}=\hbar K_{z}^{2} \gamma_{A}^{2} / 2 \gamma^{3} m_{e}=8 \hbar \gamma k_{0}^{2} / \gamma_{A}^{2} m_{e}$. An expression analogous to (12) can be derived for the static wiggler case [22]. 
For $\Omega \approx v_{0} K_{z}$ and $\omega_{0}^{2} \gg \omega_{p e}^{2}$, we have $D_{A}(\Omega, \mathbf{K}) \approx$ $4 c^{2} K_{z} k_{0}$ in Eq. (9), so that

$$
D_{L}(\Omega, \mathbf{K}) D_{A}\left(\omega_{-}, \mathbf{k}_{-}\right)=\frac{2 \omega_{p e}^{2} c^{2} K_{z} k_{0}}{\gamma^{3}} a_{0}^{2} .
$$

Setting $\Omega=v_{0} K_{z}+\Omega_{p}+i \Gamma$, where the real part $\Gamma_{R}$ of $\Gamma$ is the growth rate, and choosing $K_{z}$ and $\Omega$ so that $D_{A}\left(\omega_{-}, \mathbf{k}_{-}\right)=$ $D_{L}(\Omega, \mathbf{K})=0$ for $\Gamma=0$, we obtain $D_{L}=-2 i \Omega_{p} \Gamma+\Gamma^{2}$ and $D_{A}=2 i \Gamma\left(\omega_{0}-v_{0} K_{z}-\Omega_{p}\right)+\Gamma^{2} \approx 2 i \Gamma\left(\omega_{0}-v_{0} K_{z}\right) \approx$ $2 i \Gamma c K_{z}$ for $K_{z} \gg k_{0}$ and $v_{0} \approx-c$. Hence, inserting the expressions for $D_{L}$ and $D_{A}$ into Eq. (13), we have

$$
\Gamma^{2}\left(2 \Omega_{p}+i \Gamma\right)=\frac{\omega_{p e}^{2} c k_{0}}{\gamma^{3}} a_{0}^{2} .
$$

For $|\Gamma| \gg \Omega_{p}$, we are in the Compton regime where the ponderomotive potential of the laser dominates, with the growth rate of the instability given by

$$
\Gamma_{R}=\frac{\sqrt{3}}{2} \frac{\left(\omega_{p e}^{2} c k_{0}\right)^{1 / 3}}{\gamma} a_{0}^{2 / 3} .
$$

For this case, the quantum recoil effect is negligible [18]. On the other hand, for $|\Gamma| \ll\left|\Omega_{p}\right|$, we have an instability with the growth rate

$$
\Gamma_{R}=\left(\frac{\omega_{p e}^{2} c k_{0}}{2 \gamma^{3} \Omega_{p}}\right)^{1 / 2} a_{0} .
$$

Clearly, since $\Omega_{p}$ is in the denominator, the quantum recoil effect leads to a decrease of the growth rate. Comparing Eqs. (15) and (16), we find that the limiting amplitude between the two regimes is given by $a_{0}=a_{\text {crit }}$, where

$$
a_{\mathrm{crit}}=\left(\frac{27 \gamma^{3} \Omega_{p}^{3}}{8 \omega_{p e}^{2} c k_{0}}\right)^{1 / 2} .
$$

Equation (15) is valid for $a_{0} \gg a_{\text {crit }}$ and Eq. (16) for $a_{0} \ll a_{\text {crit }}$. In the Raman regime $k_{0} \ll k_{0 \text {,crit }}$, Eq. (16) gives the growth rate $\Gamma_{R} \approx\left(\omega_{p e} c k_{0} / 2 \gamma^{3 / 2} \gamma_{A}\right)^{1 / 2} a_{0}$, while in the quantum regime $k_{0} \gg k_{0 \text {,crit }}$, Eq. (16) yields $\Gamma_{R} \approx\left(\omega_{p e}^{2} \gamma_{A}^{2} m_{e} c / 16 \gamma^{4} \hbar k_{0}\right)^{1 / 2} a_{0}$. In Fig. 1, we have illustrated different regimes for the FEL instability, including the quantum and Raman regimes for small amplitude wiggler fields, and the Compton regime for large amplitudes. The transition from the quantum to the Compton regime in Fig. 1 corresponds to the quantum FEL parameter [8] $\bar{\rho}=\rho m_{e} c \gamma_{r} / \hbar K_{z}$, going from smaller to larger values than unity, where $\rho=\left(a_{0} \omega_{p e} / 4 c k_{0}\right) / \gamma_{r}$ is the classical

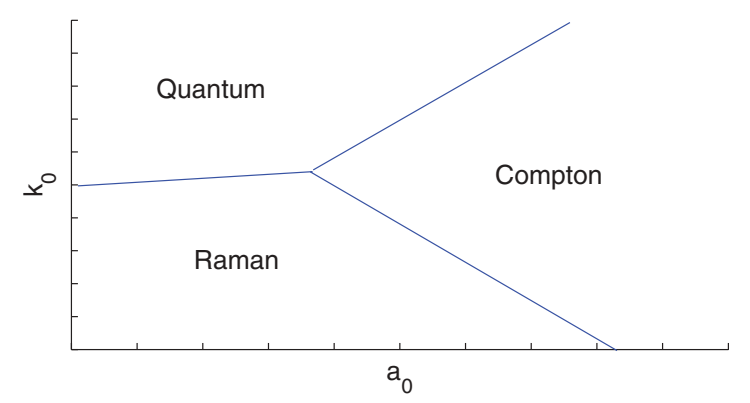

FIG. 1. (Color online) Schematic picture of different regimes for the FEL instability, showing the quantum regime $k_{0}>k_{0, \text { crit }}$, the Raman regime $k_{0}<k_{0 \text {, crit }}$, and the Compton regime $a_{0}>a_{\text {crit }}$.

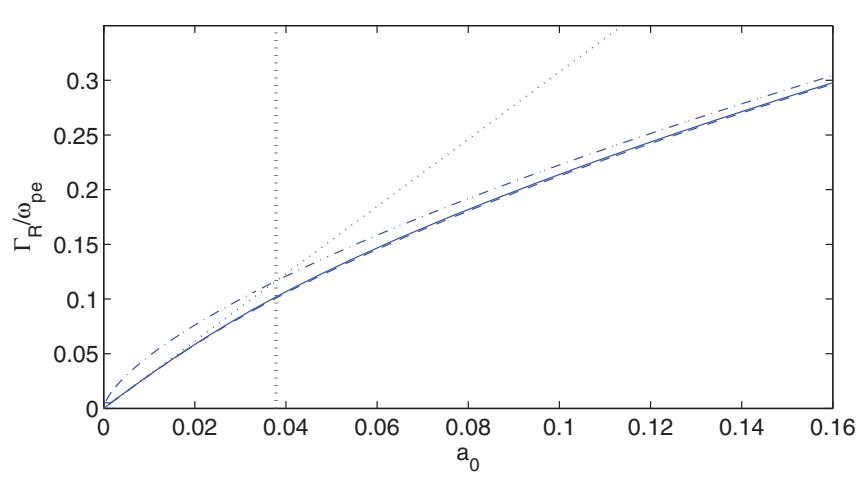

FIG. 2. (Color online) The maximum growth rate as a function of $a_{0}$ for $K_{\perp}=0$ and $\gamma=5$, using the full dispersion relation (8) (solid curve), and the approximations (14)-(16) (dashed, dashed-dotted, and dotted curves, respectively). The vertical dotted line indicates $a_{0}=a_{\text {crit }}=0.038$.

Bonifacio-Pellegrini-Narducci (BPN) parameter [23], and $\gamma_{r}$ the resonant energy in $m_{e} c^{2}$ units.

Numerical results. For illustrative purposes, we choose a beam density $n_{0}=2.2 \times 10^{22} \mathrm{~m}^{-3}$, giving $\omega_{p e}=8.37 \times$ $10^{12} \mathrm{~s}^{-1}, a_{0}=0.15$, and a wiggler wavelength of $\lambda_{0}=1 \mu \mathrm{m}$, giving $k_{0}=2 \pi / \lambda_{0}=6.36 \times 10^{6} \mathrm{~m}^{-1}$ [17]. For $\gamma=5$ one has $k_{0, \text { crit }}=1.27 \times 10^{7} \mathrm{~m}^{-1}>k_{0}$, so that the plasma oscillation effect dominates over the quantum recoil effect. Figure 2 displays the growth rate as a function of $a_{0}$, obtained from the exact dispersion relation (8) and from the approximations (14)-(16). We note that the growth rate obtained from (14) agrees very well with the one obtained from (8). Since $a_{\text {crit }}=0.038<a_{0}$, the ponderomotive force dominates over the plasma and quantum oscillations, so that Eq. (15) can be used to calculate the growth rate, giving $\Gamma_{R}=2.6 \times 10^{12} \mathrm{~s}^{-1}$ and an interaction length scale $c / \Gamma_{R} \approx 0.1 \mathrm{~mm}$.

On the other hand, due to the quantum recoil effect, the gain can rapidly decrease for higher values of $\gamma$. Using the same parameters as above but $\gamma=36$ [17], we have $k_{0 \text {, crit }}=1.07 \times$ $10^{6} \mathrm{~m}^{-1}<k_{0}$, so that the quantum recoil effect dominates the beam oscillations. Here we have $a_{\text {crit }}=1.6 \gg a_{0}$, so that Eq. (16) can be used to estimate the growth rate, which gives $\Gamma_{R}=1.5 \times 10^{11} \mathrm{~s}^{-1}$ and an interaction length $c / \Gamma_{R}=2 \mathrm{~mm}$. For this case, the expression (15) overestimates the growth rate to $\Gamma_{R} / \omega_{p e} \approx 3.5 \times 10^{11} \mathrm{~s}^{-1}$, giving $c / \Gamma_{R} \approx 1 \mathrm{~mm}$.

The instability of oblique scattering is shown in Fig. 3 for resonant radiation wave numbers $k_{z-}=k_{0}-K_{z}$ and $k_{\perp_{-}}=$ $-K_{\perp}$, obeying the resonance condition $\left(K_{z}-2 \gamma_{0}^{2} k_{0}\right)^{2}+$ $\gamma_{0}^{2} K_{\perp}^{2}=4 \gamma_{0}^{4} k_{0}^{2}$ derived above. The growth rate, deduced from Eq. (14), is almost independent of the radiation wave numbers for $\gamma=5$, where quantum recoil effects are unimportant. For $\gamma=36$, there is a significant decrease of the growth rate for larger radiation wave numbers due to quantum recoil effects, primarily in the parallel direction. For too wide electron beams, it could lead to a broadband radiation emission due to oblique scattering, while for narrow electron beams this is prevented due to a decrease of the possible interaction length in the perpendicular direction.

Discussions. Summarizing, we have presented a nonlinear model for relativistic quantum X-ray FELs, using a collective Klein-Gordon model for relativistic electrons, coupled with the 

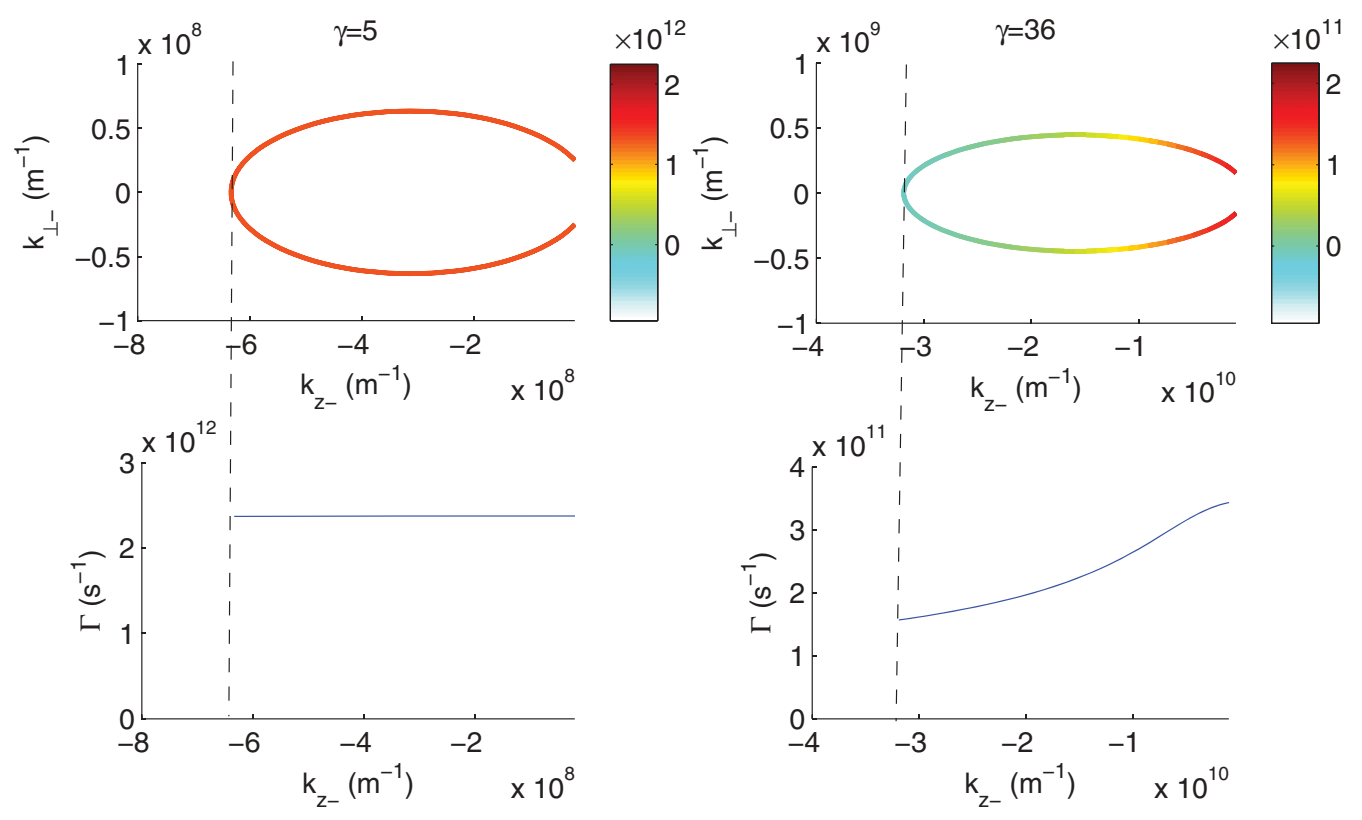

FIG. 3. (Color online) Resonant parallel and perpendicular radiation wave numbers $k_{z-}$ and $k_{\perp-}$ for $\gamma=5$ and $\gamma=36$ (top panels) with the corresponding growth rate $\Gamma\left(\mathrm{s}^{-1}\right)$ shown in color (grayscale). The bottom panels show the growth rate as a function of $k_{z-}$. (The scalings of the vertical axes are enhanced in the top panels.) The vertical bars show the location of the largest resonant wave numbers $\approx-4 k_{0} \gamma^{2} / \gamma_{A}^{2}$.

Maxwell equations for the EM fields, for an arbitrarily large amplitude laser wiggler field. We have derived a nonlinear dispersion relation for the amplification of the radiation due to scattering instability in three space dimensions. It is found that quantum recoil effects can decrease the growth rate of the resonant instability, primarily parallel to the beam direction, increasing the interaction length over which the radiation amplification occurs. The present study has assumed that the coherence of the relativistic electron beam and its transverse emittance [24] are unaffected by the quantum effects over the scale length of out interest. The quantum effect could be important if the thermal de Broglie wavelength $\lambda_{\text {th }}=\hbar \sqrt{2 \pi / m_{e} k_{B} T_{e}}$ is comparable to the interparticle distance $n_{0}^{-1 / 3}$ [25]. For $n_{0}=2.2 \times 10^{22} \mathrm{~m}^{-3}$ this happens only for $T_{e}<4 \mathrm{~K}$ when the beam electrons are Fermi degenerate. At room temperature and above, the thermal effects clearly dominate over the quantum degeneracy effects on the beam emittance. On the other hand, quantum diffusion due to spontaneous photon emission could lead to an increase of the energy spread of the electron beam. To estimate the relative energy spread, we use the formula [26] $\Delta \gamma^{2}=(14 / 15) \lambda_{C} r_{e} \gamma^{4} k_{0}^{3} a_{0}^{2} F\left(a_{0}\right) \Delta z$, where $F\left(a_{0}\right) \approx 1$ for $a_{0}<1, r_{e} \approx 2.8 \times 10^{-15} \mathrm{~m}$ is the classical electron radius, and $\Delta z$ is the interaction distance, which can be taken to be 10 $e$-foldings, $\Delta z=10 \times c / \Gamma_{R}$. For the case $\gamma=5$ above we obtain the energy spread $\Delta \gamma=6 \times 10^{-5}$, while for $\gamma=36$ we have the relatively large value $\Delta \gamma=0.014$, which might influence the performance of the FELs.

Acknowledgment. This work was supported by the Deutsche Forschungsgemeinschaft (DFG) through the Project SH21/3-2 of the Research Unit 1048.
[1] E. Hand, Nature (London) 461, 708 (2009).

[2] S. H. Glenzer et al., Phys. Rev. Lett. 98, 065002 (2007); P. Neumayer et al., ibid. 105, 075003 (2010); S. H. Glenzer and R. Redmer, Rev. Mod. Phys. 81, 1625 (2009).

[3] A. V. Andreev, JETP Lett. 72, 238 (2000); G. Mourou et al., Rev. Mod. Phys. 78, 309 (2006); P. K. Shukla and B. Eliasson, ibid. 83, 885 (2011); M. Marklund and P. K. Shukla, ibid. 78, 591 (2006).

[4] J. M. J. Madey, J. Appl. Phys. 42, 1906 (1971).

[5] F. A. Hopf et al., Phys. Rev. Lett. 37, 1342 (1976); T. Kwan et al., Phys. Fluids 20, 581 (1977); N. M. Kroll and W. A. McMullin, Phys. Rev. A 17, 300 (1978).

[6] R. Bonifacio et al., Nucl. Instrum. Methods Phys. Res., Sect. A 237, 168 (1985).

[7] C. B. Schroeder, C. Pellegrini, and P. Chen, Phys. Rev. E 64, 056502 (2001).
[8] R. Bonifacio et al., Nucl. Instrum. Methods Phys. Res., Sect. A 543, 645 (2005).

[9] R. Bonifacio et al., Europhys. Lett. 69, 55 (2005).

[10] Y. T. Yan and J. M. Dawson, Phys. Rev. Lett. 57, 1599 (1986); C. Joshi et al., IEEE J. Quantum Electron. QE-23, 1571 (1987).

[11] J. K. McIver and M. V. Federov, Sov. Phys. JETP 49, 1012 (1979); I. V. Smetanin, Laser Phys. 7, 318 (1997).

[12] G. Preparata, Phys. Rev. A 38, 233 (1988).

[13] H. K. Avetissian and G. F. Mkrtchian, Phys. Rev. E 65, 046505 (2002); Nucl. Instrum. Methods Phys. Res., Sect. A 483, 548 (2002); Phys. Rev. ST Accel. Beams 10, 030703 (2007); H. K. Avetissian, Relativistic Nonlinear Electrodynamics (Springer, New York, 2006).

[14] R. Bonifacio, L. De Salvo, P. Pierini, N. Piovella, and C. Pellegrini, Phys. Rev. Lett. 73, 70 (1994). 
[15] R. Bonifacio et al., Nucl. Instrum. Methods Phys. Res., Sect. A 593, 69 (2008).

[16] A. Serbeto et al., Phys. Plasmas 15, 013110 (2008).

[17] N. Piovella, M. M. Cola, L. Volpe, A. Schiavi, and R. Bonifacio, Phys. Rev. Lett. 100, 044801 (2008); M. M. Cola et al., Nucl. Instrum. Methods Phys. Res., Sect. A 593, 75 (2008).

[18] A. Serbeto, L. F. Monteiro, K. H. Tsui, and J. T. Mendonça, Plasma Phys. Controlled Fusion 51, 124024 (2009).

[19] T. Takabayasi, Prog. Theor. Phys. 9, 187 (1953).

[20] B. Eliasson and P. K. Shukla, Phys. Rev. E 83, 046407 (2011).

[21] J. F. Drake et al., Phys. Fluids 17, 778 (1974).

[22] For a static wiggler [7] we would have $\omega_{0}=0$ in the expression for $D_{A}$, with the result $K_{z}=2 k_{0} \gamma^{2} / \gamma_{A}^{2}$ and $\omega_{-}=$
$-\Omega=2 \gamma^{2} c k_{0} / \gamma_{A}^{2}$, which differs by a factor of 2 from the electromagnetic wiggler. Using $K_{z}=2 \gamma_{0}^{2} k_{0} / \gamma_{A}^{2}$ in Eq. (11), we obtain, analogously to (12), $k_{0, \text { crit }}=\left(\omega_{p e} \gamma_{A}^{3} m_{e} / 2 \gamma^{5 / 2} \hbar\right)^{1 / 2}$, with $\Omega_{p}=\omega_{p e} \gamma_{A} / \gamma^{3 / 2}$ for $k_{0} \ll k_{0, \text { crit }}$ and $\Omega_{p}=2 \hbar \gamma k_{0}^{2} / \gamma_{A}^{2} m_{e}$ for $k_{0} \gg k_{0, \text { crit }}$.

[23] R. Bonifacio et al., Opt. Commun. 50, 373 (1984).

[24] Z. Huang and K.-J. Kim, Phys. Rev. ST Accel. Beams 10, 034801 (2007)

[25] B. J. Claessens, S. B. van der Geer, E. J. D. Vredenbregt, and O. J. Luiten, Phys. Rev. Lett. 95, 164801 (2005).

[26] E. L. Saldin, E. A. Schneidmiller, and M. V. Yurkov, Nucl. Instrum. Methods Phys. Res., Sect. A 381, 545 (1996). 